\title{
Vermeidung postoperativer Wundinfektionen
}

\author{
J. Pochhammer ${ }^{1}$, J.-C. Harnoss², P. Walger ${ }^{3}$, C.-D. Heidecke ${ }^{4}$, S. Maier ${ }^{5}$, A. Kramer ${ }^{6}$ \\ ${ }^{1}$ Klinik für Allgemein-, Viszeral- und Thoraxchirurgie, Marienhospital Stuttgart, Vinzenz von Paul Kliniken \\ ${ }^{2}$ Klinik für Allgemein-, Viszeral- und Transplantationschirurgie, Universitätsklinikum Heidelberg \\ ${ }^{3}$ Internistische Intensivmedizin und Infektiologie, Johanniterkrankenhaus, Johanniterkliniken Bonn \\ ${ }^{4}$ Klinik und Poliklinik für Chirurgie, Allg. Chirurgie, Viszeral-, Thorax- und Gefäßchirurgie, Universitätsmedizin Greifswald \\ ${ }^{5}$ Chirurgische Klinik I, Klinikum Kaufbeuren, Kliniken Ostallgäu-Kaufbeuren \\ ${ }^{6}$ Institut für Hygiene und Umweltmedizin, Universitätsmedizin Greifswald
}

Die Vermeidung postoperativer Wundinfektionen ist ethische Verantwortung und zugleich gesetzliche Verpflichtung. Sie stellt eine permanente Herausforderung für alle an der chirurgischen Versorgung beteiligten Ärzte und Pflegekräfte dar. Die Etablierung und Umsetzung einer interdisziplinären Multibarrierenstrategie ist für den Arzt eine Führungsaufgabe, die hohe Sachkenntnis und Konsequenz erfordert, um bei allen Beteiligten für die erforderliche kontinuierliche Compliance zu sorgen.

Aufgrund der fortlaufenden Veränderung der Studienlage sind die Identifizierung von Risikofaktoren und die Überprüfung etablierter Präventionsmaßnahmen in regelmäßigen Abständen erforderlich. Risikofaktoren und die Maßnahmen zur Prävention lassen sich der prä-, peri-, intra- und postoperativen Phase zuordnen. Die Maßnahmen einschließlich der erforderlichen Rahmenbedingungen werden in dieser Übersicht einer Bewertung gemäß den vom Robert Koch-Institut (RKI) empfohlenen Evidenzkategorien unterzogen, um Handlungsanweisungen für die Praxis abzuleiten. Durch Kontrolle der Einhaltung der in einem SSI-Bündel (Surgical Site Infection) herausgehobenen Maßnahmen mittels Checkliste kann das kollektive Zusammenwirken vervollständigt werden.

\section{Einleitung}

Der Schutz des Patienten vor postoperativen Wundinfektionen (Surgical Site Infections, SSI) ist ein wesentliches Qualitätsmerkmal für das operative Behandlungsergebnis und entscheidend für die Patientenzufriedenheit. Zusätzlich müssen die Mitarbeiter vor berufsbedingten Infektionen geschützt werden.

Rangierten 1995 SSI an deutschen Akutkrankenhäusern mit ca. 16\% noch an 3. Stelle der nosokomialen Infektionen nach nosokomialer Pneumonie und Harnwegsinfektionen, sind sie 2011 mit $24,7 \%$ an die 1 . Stelle gerückt [1]. Da sich im Zeitraum 2010-2014 die SSI-Rate im Vergleich zum Zeitraum 2009-2012 gering reduziert hat, dürfte es sich um eine Verschiebung der Häufigkeiten handeln. Hierfür kommen v.a. folgende Ursachen in Betracht:

- Versorgung zunehmend älterer Patienten mit eingeschränkter Immunabwehr

- Eingriffe aufgrund moderner Operationstechniken, die früher nicht möglich waren

- Ausbreitung multiresistenter Erreger (MRE) einschließlich der damit verbundenen fehlenden Effektivität der perioperativen Antibiotikaprophylaxe (PAP), sofern das Vorkommen des MRE nicht bekannt ist
Durch Fall-Kontroll-Studien konnte gezeigt werden, dass Patienten mit SSI eine 60\% höhere Wahrscheinlichkeit haben, auf der Intensivstation behandelt zu werden, eine 2-fach höhere Wahrscheinlichkeit haben, zu sterben, sowie die 5-fach höhere Wahrscheinlichkeit einer erneuten

\section{Abkürzungen}

AWMF Arbeitsgemeinschaft der Wissenschaftlichen Medizinischen Fachgesellschaften

ASA-Score Klassifikation der American Society of Anesthesiologists

BfArM Bundesinstitut für Arzneimittel und Medizinprodukte

CDC Centers for Disease Control and Prevention

ESBL erweitertes Spektrum $\beta$-Laktamase

KRINKO Kommission für Krankenhaushygiene und Infektionsprävention des Robert Koch-Instituts

MP Medizinprodukt

MRE multiresistenter Erreger

MRSA multiresistenter Staphylococcus aureus

NNIS-Score National nosocomial Infections Surveillance Score, Normogramm zur Risikoabschätzung von SSI, Onlinekalkulation unter www.ohri.ca/SSI_risk_index

PAP perioperative Antibiotikaprophylaxe

RKI Robert Koch-Institut

SSI Surgical Site Infection (Wundinfektion)

ZVK zentraler Venenkatheter 
Krankenhausaufnahme. Durchschnittlich entstehen durch eine SSI Kosten von ca. 3000 Euro, die Aufenthaltsdauer in der Klinik verlängert sich um ca. 6,5 d. Hochgerechnet bedeutet das eine Mehrbelastung für die Versicherungssysteme von fast 300 Millionen Euro und 614000 zusätzliche Krankenhausbehandlungstage pro Jahr in Deutschland.

Definition von SSI. Für SSI gibt es zahllose Definitionen. Die in der internationalen Literatur gängigste ist die der amerikanischen CDC (Centers for Disease Control and Prevention), die 1992 von Horan et al. [2] publiziert wurde (siehe Infobox „Prinzipien“).

\section{Prinzipien}

\section{Definition chirurgischer Wundinfektionen}

1. Auftreten einer Infektion innerhalb von $30 \mathrm{~d}$ nach Operation (bis 1 Jahr, wenn Implantat) und

2. Vorhandensein mindestens 1 der 3 in Tab. 1 genannten Kriterien und/oder

3. Diagnose einer Infektion durch den Operateur oder behandelnden Arzt.

\section{Tabelle 1}

Definition chirurgischer Wundinfektionen.

\begin{tabular}{|c|c|c|}
\hline $\begin{array}{l}\text { oberflächliche } \\
\text { Wundinfektion }\end{array}$ & $\begin{array}{l}\text { tiefe Infektion } \\
\text { des Schnittes }\end{array}$ & $\begin{array}{l}\text { Organbefall } \\
\text { (Körperhöhlen) }\end{array}$ \\
\hline $\begin{array}{l}\text { eitrige Sekretion aus der } \\
\text { oberflächlichen Inzision }\end{array}$ & $\begin{array}{l}\text { eitrige Sekretion aus } \\
\text { der tiefen Inzision }\end{array}$ & $\begin{array}{l}\text { eitrige Sekretion aus einer } \\
\text { tiefen Drainage }\end{array}$ \\
\hline $\begin{array}{l}\text { Erregerisolierung aus } \\
\text { aseptisch entnommenem } \\
\text { Material }\end{array}$ & $\begin{array}{l}\text { spontane Dehiszenz der } \\
\text { tiefen Inzision oder Er- } \\
\text { öfnung durch den } \\
\text { Operateur, wenn der } \\
\text { Patient mindestens } 1 \text { der } \\
\text { folgenden Symptome hat: } \\
\text { Fieber }\left(>38^{\circ} \mathrm{C}\right) \text {, lokalisierten } \\
\text { Schmerz oder Druck- } \\
\text { empfindlichkeit }\end{array}$ & $\begin{array}{l}\text { Erregerisolierung aus } \\
\text { aseptisch entnommenem } \\
\text { Material von Flüssigkeit } \\
\text { oder Gewebe im eigent- } \\
\text { lichen Operationsgebiet }\end{array}$ \\
\hline $\begin{array}{l}\text { mindestens } 1 \text { der } \\
\text { Symptome: Schmerz, } \\
\text { Druckempfindlichkeit, } \\
\text { lokalisierte Schwellung, } \\
\text { Rötung oder Überwärmung } \\
\text { und Eröffnung der Wunde }\end{array}$ & $\begin{array}{l}\text { Abszess oder andere } \\
\text { Anzeichen für eine Infektion, } \\
\text { festgestellt bei direkter } \\
\text { Untersuchung, während } \\
\text { einer Reoperation oder bei } \\
\text { histopathologischer oder } \\
\text { radiologischer Untersuchung }\end{array}$ & $\begin{array}{l}\text { Abszess oder andere } \\
\text { Anzeichen für eine Organ-I } \\
\text { Körperhöhleninfektion, } \\
\text { festgestellt bei direkter } \\
\text { Untersuchung, während } \\
\text { einer Reoperation oder } \\
\text { bei histopathologischer } \\
\text { oder radiologischer Unter- } \\
\text { suchung }\end{array}$ \\
\hline
\end{tabular}

\section{Risikofaktoren für SSI}

Die Tab. 2 gibt einen Überblick über Risikofaktoren, die v.a. bei orthopädischen und viszeralchirurgischen Eingriffen identifiziert wurden. Dazu kommt natürlich bei jedem Patienten die individuelle Disposition als unwägbarer Faktor hinzu.

\section{Präoperative Präventionsmaßnahmen}

\section{Überblick}

Die Tab. 3 zeigt die präoperativen Präventionsmaßnahmen im Überblick. Bei der vorgeschlagenen Evidenz werden die von der Kommission für Krankenhaushygiene und Infektionsprävention (KRINKO) definierten Kategorien zugrunde gelegt (Tab.4); sie kann im Einzelfall aufgrund neuerer Studien davon abweichen [3].

\section{Erläuterung der Maßnahmen}

\section{- Aufbereitung von Medizinprodukten}

Aufgrund der Bedeutung der qualitätsgesicherten Aufbereitung von Medizinprodukten (MP) für die Patientensicherheit und wiederholt festgestellter Mängel muss bereits vor der Anschaffung eines MP die Möglichkeit der Aufbereitung überprüft werden. Der Betreiber muss für jedes MP das Verfahren der Aufbereitung schriftlich festlegen und bei vom Hersteller begrenzter Anzahl von Aufbereitungen diese dokumentieren. Die Aufbereitung ist „mit geeigneten validierten Verfahren so durchzuführen, dass der Erfolg dieser Verfahren nachvollziehbar gewährleistet ist" [4]. Grundlage für die ordnungsgemäße Aufbereitung ist die gemeinsame Empfehlung der KRINKO und des Bundesinstituts für Arzneimittel und Medizinprodukte (BfArM) [5].

Für den leitenden Chirurgen ergeben sich folgende

\section{Konsequenzen:}

- Sein mit der Hygiene beauftragter Arzt sollte sich durch Vorlage des Qualitätsmanagementhandbuchs des Aufbereiters vergewissern, dass die Aufbereitung den gesetzlichen Anforderungen entspricht. Für die fachliche Überprüfung wird er die Expertise des Krankenhaushygienikers in Anspruch nehmen.

- Für die Aufbereitung innerhalb der niedergelassenen Praxis besteht die Verpflichtung zur Qualitätssicherung, es ist jedoch keine Zertifizierung erforderlich. 
Risikofaktoren für das Entstehen von SSI.

\begin{tabular}{|c|c|c|c|}
\hline präoperativ & perioperativ & eingriffsspezifisch & postoperativ \\
\hline $\begin{array}{l}\text { - } \text { ASA-Score > } 2 \\
\text { längerer präoperativer Krankenhaus- } \\
\text { - } \text { hohes Lebensalter } \\
\text { - Vorerkrankungen wie Diabetes mellitus, } \\
\text { Hyperglykämie, dialysepflichtige } \\
\text { Niereninsuffizienz, Lebererkrankung, } \\
\text { Cholestase, chronisch obstruktive Lun- } \\
\text { generkrankung, Gefäßerkrankungen } \\
\text { - Infektion anderer Lokalisation } \\
\text { - Infektion/Kolonisation mit MRE } \\
\text { - nasale Kolonisation mit Staphylococcus } \\
\text { aureus/MRSA } \\
\text { - Mangelernährung } \\
\text { - Adipositas } \\
\text { - Nikotin-/Alkoholabusus } \\
\text { - fortgeschrittene maligne Grund- } \\
\text { erkrankung } \\
\text { - Vorbestrahlung } \\
\text { - Anämie } \\
\text { - Granulozytopenie } \\
\text { - Thrombozytose } \\
\text { - Koagulopathie }\end{array}$ & $\begin{array}{l}\text { - unsachgemäße Operations- } \\
\text { feldvorbereitung } \\
\text { - falsche Händedesinfektion } \\
\text { - unwirksame PAP } \\
\text { - verzögerter Operationszeitpunkt } \\
\text { - Hypothermie } \\
\text { - Hypoxie } \\
\text { - bakterienpermeable Schutz- } \\
\text { - kleidung } \\
\text { - Handschuhperforation } \\
\text { - respiratorische Infektion des } \\
\text { Operationsteams }\end{array}$ & $\begin{array}{l}\text { - } \text { hoher NNIS-Score } \\
\text { - Operationsdauer } \\
\text { - } \text { Art des Eingriffs (z. B. Notfall- vs. } \\
\text { Elektiveingriff, Kontaminations- } \\
\text { grad, Rezidiveingriff) } \\
\text { - Operationstechnik } \\
\text { - Blutstillung } \\
\text { - Fremdkörperimplantation } \\
\text { - Erfahrung des Operateurs }\end{array}$ & $\begin{array}{l}\text { - Schmerz } \\
\text { - unsachgemäße Wundversor- } \\
\text { gung } \\
\text { - Drainagen } \\
\text { - verzögerte enterale Ernährung } \\
\text { - antibiotische Behandlung > 1 d } \\
\text { - postoperative invasive Maß- } \\
\text { nahmen, die mit Bakteriämie } \\
\text { einhergehen } \\
\text { - Unterkühlung }\end{array}$ \\
\hline
\end{tabular}

- Krankenhäuser, die für sich selbst und zusätzlich für andere Einrichtungen aufbereiten, müssen nicht zertifiziert und nicht beim Deutschen Institut für medizinische Dokumentation und Information gemeldet sein. Rechtlich ist die Situation des externen Aufbereiters aufgrund der Definition des sog. Inverkehrbringens von MP allerdings nicht eindeutig, sodass einige Juristen eine Zertifizierung empfehlen und viele Krankenhäuser sich daran orientieren.

- MP, die bestimmungsgemäß die Haut oder Schleimhaut durchdringen, an Organen zur Anwendung bzw. in Kontakt mit Blut und Wunden kommen, werden als kritisch eingestuft. Zusätzlich wird die erforderliche Aufbereitung eingestuft. Für MP der Kategorie „Kritisch A“ (z.B. Wundhaken) wird ein Qualitätsmanagement gefordert, für „Kritisch B“ (z.B. Laparoskopietrokare) muss eine anerkannte Ausbildung des Aufbereiters nachgewiesen werden. Für die Kategorie „Kritisch C“ (z.B. Katheter für endoskopische retrograde Cholangiopankreatikografie) muss zusätzlich die Aufbereitung und das Qualitätsmanagementsystem durch eine anerkannte Stelle zertifiziert sein.

\section{- Händehygiene}

\section{Durch Mängel in der hygienischen Händedesinfektion}

können z.B. MRE übertragen werden. Diese können den Orogastrointestinaltrakt des Patienten kolonisieren und Ursache einer SSI werden. Das Einbringen nosokomialer Erreger in die Wunde bei nicht korrekter Versorgung sekundär heilender Wunden ist ein anderes Beispiel.

Bei den im Rahmen der nationalen Händedesinfektionskampagne 2014 erhobenen Daten wurde deutlich, dass trotz einer Compliance von durchschnittlich $73 \%$ vor Intervention noch Verbesserungspotenzial besteht. Verbesserungen sind v.a. durch Schulung und Training, ausreichende Anzahl von Desinfektionsmittelspendern, Überwachung des Verbrauchs an Händedesinfektionsmittel, In-Prozess-Überwachung und Einbeziehung des Patienten in die Händehygiene erreichbar.

Künstliche Fingernägel waren wiederholt Ursache von Ausbrüchen. Fingerringe behindern die Durchführung der Händedesinfektion, sind mit erhöhter Nachweisrate 


\section{Perioperative Medizin}

\section{Tabelle 3}

\section{Präoperative Präventionsmaßnahmen.}

\begin{tabular}{|l|l|}
\hline Maßnahmen & Evidenzgrad \\
\hline Basishygiene & \\
\hline $\begin{array}{l}\text { qualitätsgerechte Aufbereitung von } \\
\text { Medizinprodukten }\end{array}$ & IA/IV \\
\hline hygienische Händedesinfektion & IA \\
\hline Verbot künstlicher Fingernägel und Fingerringe & IB/IV \\
\hline Hautschutz & ॥ \\
\hline fachspezifische Präventionsmaßnahmen & \\
\hline
\end{tabular}

\section{Sanierung bestehender Infektionen vor elektiven IA} Eingriffen

\begin{tabular}{|l|l|}
\hline Einstellung des Rauchens & IB \\
\hline kurze präoperative Verweildauer & IB \\
\hline $\begin{array}{l}\text { Korrektur metabolischer Abweichungen } \\
\text { bei elektiven Eingriffen }\end{array}$ & $\|$ \\
\hline Gewichtsreduktion bei elektiven Eingriffen & $\|$ \\
\hline Probiotika (indikationsabhängig) & $\|$ \\
\hline
\end{tabular}

Screening und Dekolonisierung von Staphylococcus II aureus bei besonderer Gefährdung

risikoadaptiertes Screening auf multiresistente III Staphylococcus aureus und Dekolonisierung

risikoadaptiertes Screening auf vancomycinIII resistente Enterokokken

risikoadaptiertes Screening auf 3- und 4-multiIII resistente gramnegative Erreger bei Operationen mit indizierter perioperativer Antibiotikaprophylaxe

nosokomialer Erreger an den Händen assoziiert und begünstigen die Handschuhperforation.

\section{Tabelle 4}

Übersicht über die Evidenzgrade des Robert Koch-Intituts.

\section{Kategorie Erläuterung}

\begin{tabular}{|l} 
IA $\begin{array}{l}\text { Diese Empfehlung basiert auf gut konzipierten syste- } \\
\text { matischen Reviews oder einzelnen hochwertigen } \\
\text { randomisierten kontrollierten Studien. }\end{array}$ \\
\hline IB \\
$\begin{array}{l}\text { Diese Empfehlung basiert auf klinischen oder hoch- } \\
\text { wertigen epidemiologischen Studien und strengen, } \\
\text { plausiblen und nachvollziehbaren theoretischen } \\
\text { Ableitungen. }\end{array}$ \\
II \\
$\begin{array}{l}\text { Diese Empfehlung basiert auf hinweisenden Studien/ } \\
\text { Untersuchungen und strengen, plausiblen und nach- }\end{array}$ \\
III $\quad \begin{array}{l}\text { Hierzu zählen Maßnahmen, über deren Wirksamkeit } \\
\text { nur unzureichende oder widersprüchliche Hinweise } \\
\text { vorliegen, deshalb ist eine Empfehlung nicht möglich. }\end{array}$ \\
Hierzu zählen Anforderungen, Maßnahmen und \\
Verfahrensweisen, die durch allgemein geltende \\
Rechtsvorschriften zu beachten sind.
\end{tabular}

\section{- Hautschutz}

Da bereits kleinste Lazerationen der Epidermis zum Erregerreservoir werden können, ist eine glatte und gepflegte Haut Voraussetzung einer effektiven Händedesinfektion. Deshalb empfiehlt es sich, zu Arbeitsbeginn und ggf. in Arbeitspausen Hautschutzpräparate aufzutragen. Hierdurch wird der Hautzustand signifikant verbessert, ohne dass die Wirksamkeit der Händedesinfektion beeinträchtigt wird. Die Präparate sollen vom Arbeitgeber allerorts zur Verfügung gestellt werden.

\section{- Sanierung von Infektionen vor elektiven Eingriffen}

Vor elektiven Eingriffen sollen klinisch manifeste Infektionen außerhalb sowie im Operationsgebiet als Risikofaktor identifiziert und saniert werden.

\section{- Rauchen}

Rauchen

- verzögert die Wundheilung,

- reduziert die Hautdurchblutung,

- stört die Hämoglobinfunktion,

- wirkt immunsuppressiv und

- beeinträchtigt respiratorische Funktionen. 
Der positive Effekt der präoperativen Nikotinkarenz auf Morbidität und Letalität ist nachgewiesen. Es wird empfohlen, Rauchen mindestens $30 \mathrm{~d}$ vor einem elektiven Eingriff einzustellen, da erst dann das Risiko pulmonaler Komplikationen sinkt. Das Risiko von SSI scheint bereits nach 2-wöchiger Abstinenz verringert. Schon nach einer Karenz von $12-48$ h sinkt der myokardiale Sauerstoffverbrauch und damit die Inzidenz kardialer Ischämien. Daher sollte für kardial erkrankte Patienten unbedingt ein präoperativer Verzicht von 1-2d eingehalten werden. Allerdings konnte ein erhöhtes Aspirationsrisiko durch präoperativen Nikotinkonsum ausgeschlossen werden, sodass Operationen deswegen nicht abgesetzt werden müssen.

\section{- Kurze präoperative Verweildauer}

Bei elektiven abdominalchirurgischen Eingriffen steigt das SSI-Risiko mit zunehmender präoperativer Verweildauer. Auch bei orthopädischen Patienten konnte die Verweildauer als unabhängiger Risikofaktor identifiziert werden.

\section{- Korrektur metabolischer Abweichungen}

Mangelernährung ist besonders für die Prognose nach Organtransplantation sowie für die postoperative Morbidität alter Menschen relevant und bei aufschiebbaren Eingriffen 7-14 d präoperativ auszugleichen. Ob das auch für Tumorerkrankungen zutrifft, ist offen, weil keine Parameter bekannt sind, in welchem Ausmaß das Fortschreiten der onkologischen Grunderkrankung bei Verschieben der Operation zu befürchten ist und fraglich ist, ob und in welcher Zeit der Ernährungszustand verbessert werden kann.

Als Parameter für das Präscreening von Risikopatienten werden ein Body-Mass-Index $<20 \mathrm{~kg} / \mathrm{m}^{2}$, Gewichtsverlust innerhalb der letzten 3 Monate, reduzierte Nahrungsaufnahme in der letzten Woche und schwere Erkrankung empfohlen.

Bei Diabetes mellitus wird prä- und postoperativ die engmaschige Kontrolle des Blutglukosespiegels mit Vermeidung von Werten > $200 \mathrm{mg} / \mathrm{dl}$ bzw. > 11,1 mmol/l empfohlen.

Obwohl Bluttransfusionen ein unabhängiger Risikofaktor für SSI sind, sollte eine präoperative Anämie ausgeglichen werden.

\section{- Gewichtsreduktion}

Bei Adipositas Grad II und III ist eine Gewichtsreduktion für Eingriffe zu erwägen, bei denen der Operationserfolg von der Körpermasse beeinflusst wird (z. B. Narbenhernie).

\section{- Probiotika}

Aussichtsreich ist der prä- und postoperative Einsatz bis zur Stabilisierung der Darmflora. Eine Reduktion von SSI wurde bei Pankreatoduodenektomie, Lebertransplantation, Hepatektomie wegen Gallengangskarzinom und kolorektaler Chirurgie erzielt. Kontraindikationen sind Sepsis, schwere Immunsuppression und akute Pankreatitis.

\section{- Dekolonisierung von Staphylococcus aureus bzw. multiresistentem Staphylococcus aureus}

Im Unterschied zu orthopädischen und herzchirurgischen Operationen ist die nasale Kolonisation mit Staphylococcus aureus für viszeralchirurgische Operationen nicht als unabhängiger Risikofaktor für SSI gesichert. Sofern ein hoher Anteil von SSI durch Staphylococcus aureus mit zugleich kritischen Folgen verursacht wird, z.B. bei kardiochirurgischen Operationen, wird ein Screening mit nachfolgender Dekolonisierung (ggf. Dekolonisierung auch ohne Screening) empfohlen. Allerdings wurde ein präventiver Einfluss nur bei präoperativer Dekolonisation über $5 \mathrm{~d}$ erzielt. Ist bei MRSA-Nachweis kein Aufschub der Operation bis zur Dekolonisation möglich, waren Vancomycin oder Teicoplanin gegenüber $\beta$-Lactam-Antibiotika bei kardiochirurgischen Operationen überlegen.

\section{Weiterführende Info}

\section{Erregerspektrum der SSI}

Das Erregerspektrum, das für die Entstehung der Wundinfektion ursächlich ist, ist abhängig von der Art des Eingriffs. Insgesamt ist Staphylococcus aureus für etwa 30\% aller SSI verantwortlich, koagulasenegative Staphylokokken für etwa 13\%. Die verschiedenen Enterokokkentypen verursachen etwa $10 \%$ der SSI. Somit ist die Mehrheit der auslösenden Organismen grampositiv. Escherichia coli und Pseudomonas spp. werden seltener nachgewiesen, müssen aber v. a. in der Abdominalchirurgie unbedingt Berücksichtigung finden. 
n Risikoadaptiertes Screening auf vancomycinresistente Enterokokken, 3- und 4-multiresistente gramnegative Erreger

Da keine präoperative Dekolonisation möglich ist, ist ein präoperatives Screening nur dann sinnvoll, wenn mit einer erhöhten Rate von SSI durch resistente Erreger zu rechnen ist, die durch die geeignete Wahl eines noch sensiblen Antibiotikums reduziert werden könnte. Die Datenlage hierzu ist inkonsistent.

Für die USA wurde geschätzt, dass ein 30\%iger Verlust der Effektivität der PAP bei den 10 häufigsten Operationen durch MRE zu 120000 zusätzlichen Infektionen und 6300 zusätzlichen Todesfällen pro Jahr führt. In diese Schätzung wurden allerdings auch die Effekte einer durch Resistenz beeinträchtigten internistisch indizierten Prophylaxe bei Chemotherapien hämatologischer Erkrankungen miteinbezogen.

Kolorektale Operationen oder andere große abdominalchirurgische Eingriffe gehen mit den höchsten SSI-Raten einher. Es ist zu vermuten, dass die Rate vorbestehender Resistenzen einen Einfluss auf die SSI-Rate hat, wenn die PAP die Resistenzen nicht abdeckt. Aus der alleinigen Erfassung präoperativer Resistenzraten kann jedoch nicht ohne Weiteres auf die SSI-Rate geschlossen werden. Bei einer in den USA üblichen PAP mit Cefazolin plus Metronidazol vor kolorektalen Eingriffen einschließlich Appendektomien lag beispielsweise der Anteil resistenter Erreger bei 43,2\% und damit höher als die Rate an SSI.

Die Auswirkungen hoher präoperativer Resistenzraten auf das SSI-Risiko sind nur für wenige Operationen untersucht. So korrelierte die präoperative Fluorchinolonresistenzrate von Escherichia coli vor Prostatabiopsien bei einer Fluorchinolonprophylaxe mit erhöhten SSI-Raten.

Nach einem Wechsel der PAP vor Pankreatoduodenektomie (Whipple-Operation) von Cefoxitin/Metronidazol auf Piperacillin/Tazobactam kam es zur deutlichen Reduktion der SSI-Rate. Die häufigsten Erreger Enterobacter spp. und Enterococcus spp. waren dabei cefoxitinresistent. Auch bei Lebertransplantationen wurde ein positiver Zusammenhang von präoperativer ESBL-Kolonisation (ESBL: erweitertes Spektrum $\beta$-Laktamase) und SSI durch ESBL nachgewiesen.

Der Wechsel der PAP vor kardio- und gefäßchirurgischen sowie orthopädischen Eingriffen von $\beta$-Laktam-Antibiotika auf Vancomycin führte zwar zur signifikanten Reduktion von MRSA- und Enterokokkeninfektionen, die Zahl respiratorischer Infektionen stieg jedoch deutlich.
Daher kann ein genereller Wechsel der PAP zur Prophylaxe von Infektionen durch resistente Erreger ohne Nachweis der Effektivität durch kontrollierte Studien nicht empfohlen werden.

\section{Perioperative Präventionsmaßnahmen}

\section{Überblick}

Die Tab. 5 zeigt die perioperativen Maßnahmen zur Prävention von SSI im Überblick. Zur Erläuterung der Evidenzgrade siehe Tab. 4.

\section{Tabelle 5}

Perioperative Präventionsmaßnahmen.

\begin{tabular}{|l|l|}
\hline Maßnahmen & Evidenzgrad \\
\hline Basishygiene
\end{tabular}

Desinfektion patientennaher Flächen

\begin{tabular}{|l|l|}
\hline Körperreinigung des Patienten & III \\
\hline Berufs-, Bereichs- und Schutzkleidung & II/IV \\
\hline
\end{tabular}

\begin{tabular}{|l|l|}
\hline fachspezifische Präventionsmaßnahmen & \\
\hline präoperative Hautantiseptik & IA \\
\hline Verzicht auf Rasur oder Clipping & IA \\
\hline perioperative Antibiotikaprophylaxe & IA \\
\hline chirurgische Händedesinfektion & IB \\
\hline $\begin{array}{l}\text { Begrenzung der Personenanzahl und -bewegung } \\
\text { im Operationssaal }\end{array}$ & IB \\
\hline
\end{tabular}

erregerdichte Operationsabdeckung

IB

Screening des Operationsteams bei Ausbruch IB von SSI durch Staphylococcus aureus oder A-Streptokokken

Wechsel der Operationshandschuhe IB

Kombination von Darmreinigung und oraler

IB Antibiose in der Darmchirurgie

antiseptische Inzisionsfolie II

Hautversiegelung III 


\section{Erläuterung der Maßnahmen}

\section{- Bettenhygiene und Desinfektion patientennaher Flächen}

Beides kann prä- und postoperativ bei Mängeln dazu führen, dass der Patient nosokomiale Pathogene akquiriert, die eine SSI verursachen können.

\section{Körperreinigung des Patienten}

Sie wird aus allgemeinhygienischen Gründen vor der Operation oder am Tag der Operation als Ganzkörperwäsche oder Dusche empfohlen, hat aber keinen direkten Einfluss auf die SSI-Rate. Die wiederholte Körperwaschung mit antiseptischen Seifen kann die Weiterverbreitung von MRE minimieren, bei einmaliger präoperativer Waschung ist aber unabhängig von der Waschlotion kein Einfluss auf die SSI-Rate nachweisbar.

\section{n Berufs-, Bereichs- und Schutzkleidung}

Vorschriften zur Berufskleidung bewegen die Gemüter fast wie Modefragen. Aufgrund der Persistenz von Pathogenen auf Textilien und des Nachweises v.a. auf dem Arztkittel kann Berufskleidung zur Verbreitung nosokomialer Pathogene beitragen. Auch private Oberbekleidung ist nicht in der direkten Patientenversorgung zu tragen. Zu empfehlen sind Hose und kurzärmliger Kasack, um die Unterarme in die Händedesinfektion einbeziehen zu können. Bei Tätigkeiten am Patienten (z. B. bei Verbandswechsel, Abb. 1) soll der Arztkittel abgelegt werden, ggf. ist ein langärmliger Schutzkittel anzulegen. Bereichskleidung dient der Verhinderung der Ein- bzw. Ausschleppung kritischer Pathogene in bzw. aus Risikobereichen und ist nur in dem festgelegten Bereich zu tragen. Ohne Mund-Nasen-Schutz wurde bei $42 \%$ der Mitarbeiter die Abgabe von Bakterien in die Umgebung nachgewiesen, was die Bedeutung von Mund-NasenSchutz bzw. Gesichtsmaske bei aseptischem Arbeiten unterstreicht.

\section{- Präoperative Hautantiseptik}

Sie soll eine Verschleppung der residenten Hautflora in die Tiefe des Operationsfelds verhindern. Entscheidend für die Effektivität sind das initiale Einreiben des Antiseptikums für $30 \mathrm{~s}$. Die nachfolgende Benetzung soll abhängig von talgdrüsenarmer oder -reicher Haut für mindestens 1,5 oder 3 min erfolgen, wobei längere Einwirkungszeiten nicht untersucht wurden.

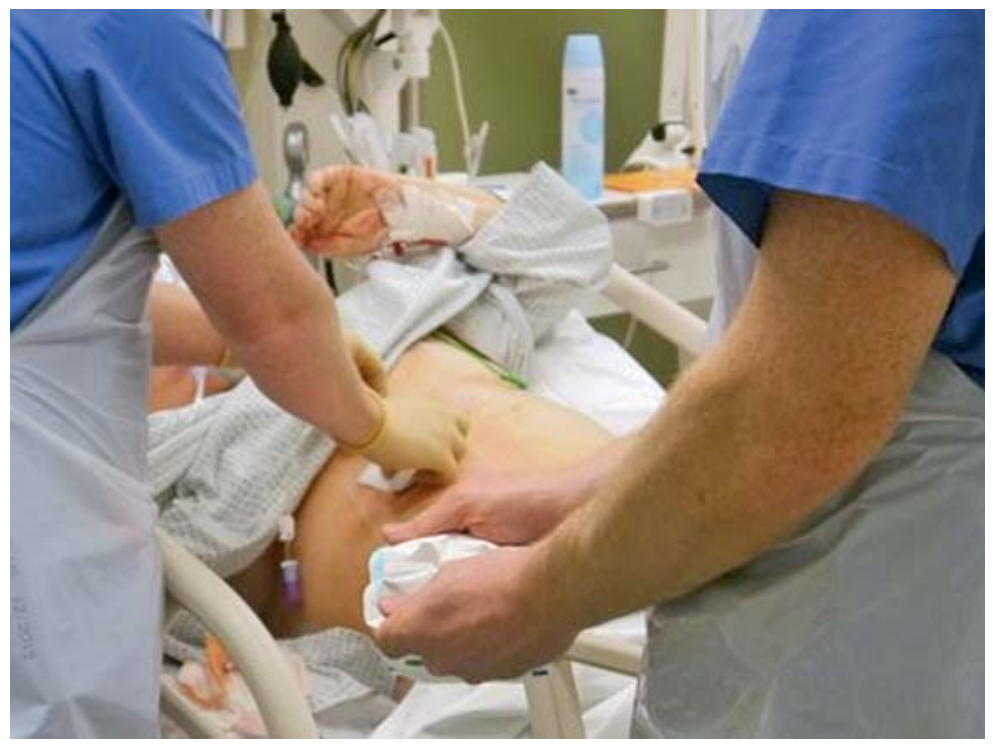

Abb. 1 - Verbandswechsel mit kurzärmeliger Bereichskleidung, Schürze und aseptischen Kautelen.

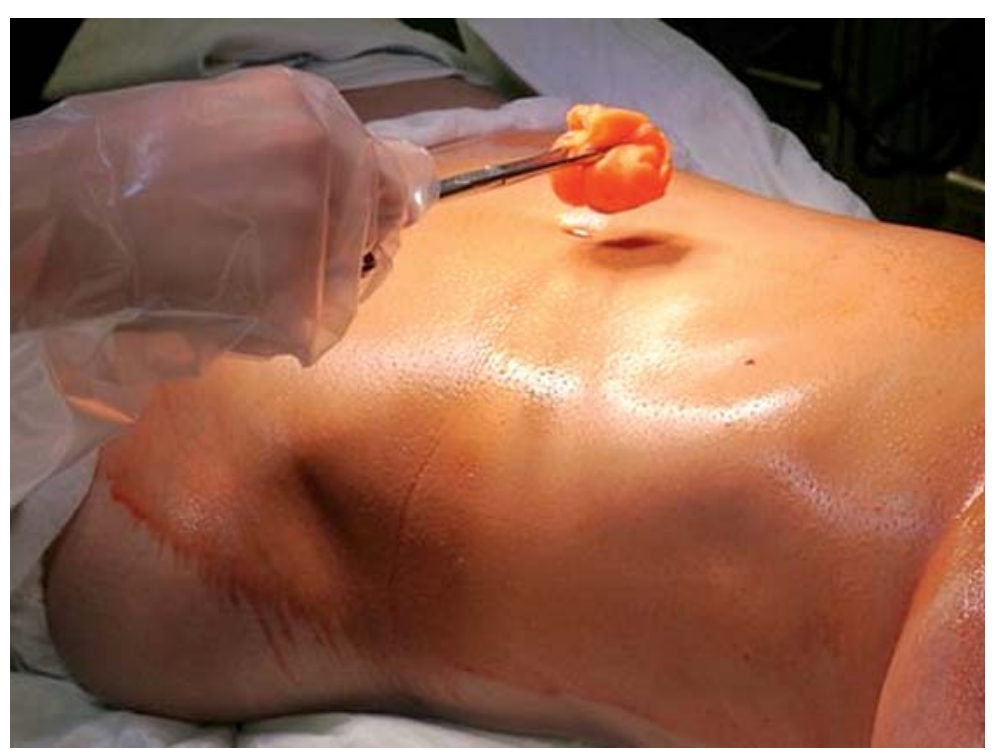

Abb. 2 - Hautantiseptik auf alkoholischer Basis mit Zusatz eines Antiseptikums mit remanenter Wirkung.

Alkoholbasierte Formulierungen sind aufgrund ihrer raschen, hohen Wirksamkeit Wirkstoff der 1. Wahl. Die wässrige Polyvinylpyrrolidon-Iod-Lösung ist wegen geringerer Wirksamkeit und des Risikos der Schilddrüsenüberfunktion nicht mehr einzusetzen. Durch Zusatz von Chlorhexidin oder Octenidin zum Alkohol wird die remanente Wirkung signifikant erhöht. Die Wirkung der zugesetzten Antiseptika setzt langsamer ein als die des Alkohols, bewirkt allerdings eine deutlich länger anhaltende Reduzierung der Hautflora (Abb. 2). 
Als Fazit aller bisherigen Studien einschließlich der Effektivität zur Prävention ZVK-assoziierter (ZVK: zentraler Venenkatheter) Blutstrominfektionen empfiehlt es sich, insbesondere bei lang dauernden Eingriffen, Alkohole mit remanentem Zusatz einzusetzen. Weiterhin ist auf eine ausreichende Einwirkzeit zu achten, ein „Trockenwischen“ vor dem Abkleben sollte vermieden werden. Es gibt auch Hinweise, dass die Anwendung von Antiseptika im zu operierenden Bereich (z.B. durch den Patienten im Rahmen eines Duschvorgangs vor dem Eingriff) sowie die mehrfache Anwendung frühzeitig vor Operationsbeginn die Hautflora deutlich reduziert.

- Die erneute Antiseptik des Wundrands vor Wundverschluss soll eine infektionspräventive Wirkung haben, hierzu besteht allerdings nur eingeschränkte Evidenz.

\section{- Hautinzision}

Die Datenlage gestattet keine Aussage, ob nach dem Hautschnitt das Skalpell getauscht werden muss. Da die Hautflora, insbesondere die der tieferen Schichten, durch die Hautantiseptik nur unvollständig eliminiert wird, kann das Skalpell bei Durchtrennung der Haut kontaminiert sein und anhaftende Erreger können in die Tiefe verschleppt werden. Allerdings war kein Einfluss auf die SSI-Rate ohne Wechsel nachweisbar.

In Anbetracht der geringen Kosten des Wechsels ist dieser solange zu empfehlen, bis die Unbedenklichkeit des Verzichts nachgewiesen ist.

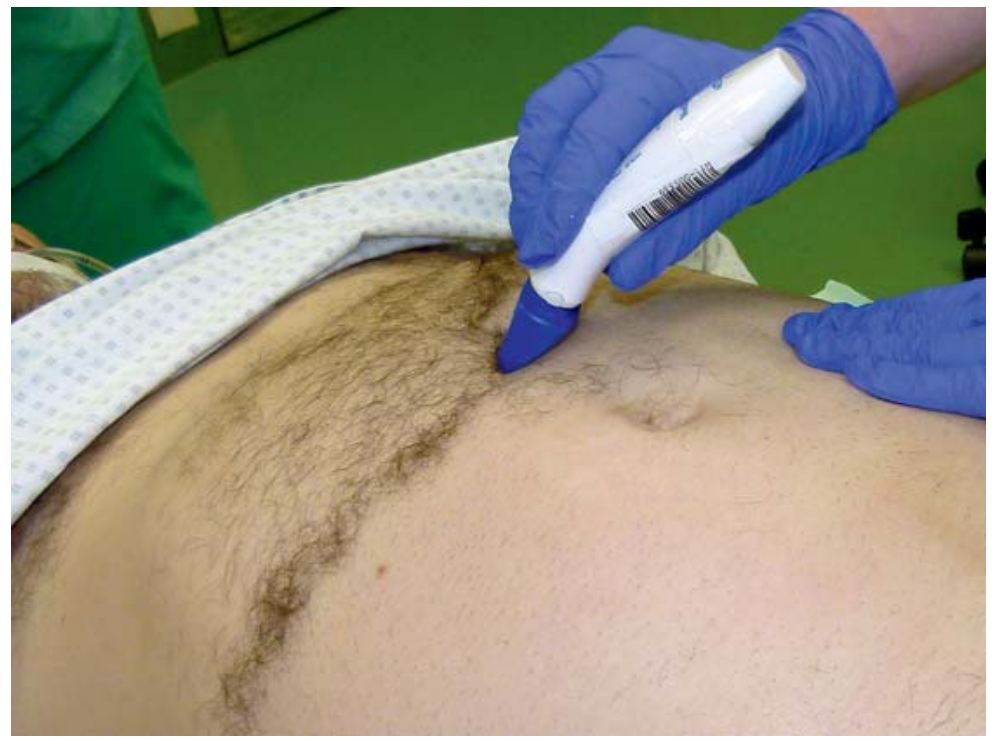

Abb. 3 - Haarentfernung mit dem Clipper.

\section{- Elektrische Rasur (Clipping) oder Verzicht}

Haare sind präoperativ nur bei operationstechnischer Notwendigkeit zu entfernen. Hierfür ist Clipping die Methode der Wahl (Abb. 3), da es die Haut mechanisch und biochemisch nicht beeinflusst. Daher kann auch der Abstand zur Operation beliebig gewählt werden. Allerdings muss eine adäquate Aufbereitung entsprechend der Herstellerangaben gewährleistet sein.

\section{- Adäquate Antibiotikaprophylaxe}

Die PAP ist wegen des Resistenzdrucks und möglicher Nebenwirkungen nur bei gesicherter Indikation gemäß den Empfehlungen der Fachgesellschaften vorzunehmen und ist indiziert bei

- hoher Erregerexposition im Operationsgebiet (Wundklassifikation sauber-kontaminiert oder kontaminiert),

- sauberen Eingriffen und Vorliegen eines zusätzlichen Risikofaktors, z.B. Notfalloperation, Hochrisikooperation, Einbau oder Ersatz von Gefäßimplantaten, Herzklappen und anderen alloplastischen Materialien,

- sauberen Eingriffen und Vorliegen patienteneigener Risiken, z.B. Immunsuppression, schwere Grunderkrankung, hoher ASA-Score, Vorbestrahlung oder Unterkühlung (Tab. 6) [6].

Ab dem Zeitpunkt des Hautschnitts muss für die Dauer der Operation ein wirksamer Blut- und Gewebespiegel des Antibiotikums gewährleistet sein. Bei starkem Blutverlust (>11) oder Operationsdauer länger als die doppelte Halbwertszeit des Antibiotikums muss eine Folgedosis verabreicht werden. Für die Auswahl sind die Wirksamkeit gegen die häufigsten Wundinfektionserreger für die jeweilige Operation, die Gewebegängigkeit und die lokale Resistenzsituation entscheidend. Bei nachgewiesenen MRE und unzureichender Möglichkeit der Eradikation sollte das Wirkspektrum der PAP den nachgewiesenen Erreger einbeziehen. $\mathrm{Zu}$ beachten ist die Dosierung gemäß Körpergewicht und bei erhöhten oder erniedrigten Verteilungsräumen.

Die Infobox „Prinzipien“ stellt Möglichkeiten der Optimierung der perioperativen Infektionsprophylaxe mit Antibiotika vor. 
Tabelle 6

Kategorisierung der Eingriffe nach Cruse.

\begin{tabular}{|c|c|c|c|}
\hline Kategorie & Beschreibung & SSI-Risiko & Beispiel \\
\hline aseptische Eingriffe & $\begin{array}{l}\text { nicht infiziertes Operationsgebiet, in dem keine Entzündung vorhanden } \\
\text { ist und weder der Respirations-, Gastrointestinal- oder Urogenitaltrakt } \\
\text { eröffnet wurde. Operative Wunden nach stumpfen, nicht penetrierenden } \\
\text { Traumata werden eingeschlossen, sofern die o.g. Kriterien erfüllt sind. }\end{array}$ & $<2 \%$ & $\begin{array}{l}\text { elektive Schilddrüsen-, Herz-, } \\
\text { Gelenkoperation }\end{array}$ \\
\hline bedingt aseptische Eingriffe & $\begin{array}{l}\text { Eingriffe, bei denen der Respirations-, Gastrointestinal- oder } \\
\text { Urogenitaltrakt unter kontrollierten Bedingungen und ohne } \\
\text { ungewöhnliche Kontamination eröffnet wird }\end{array}$ & $<10 \%$ & $\begin{array}{l}\text { Magen, Galle, Leber, Pankreas, } \\
\text { Oropharynx, Lunge, } \\
\text { Geschlechtsorgane }\end{array}$ \\
\hline kontaminierte Eingriffe & $\begin{array}{l}\text { Operationen mit einem größeren Bruch in der aseptischen Technik } \\
\text { (z. B. deutlicher Austritt von Darminhalt) sowie Eingriffe bei akuter, } \\
\text { nicht eitriger Entzündung }\end{array}$ & $5-20 \%$ & $\begin{array}{l}\text { Eingriffe mit intraoperativer } \\
\text { Hohlorganeröffnung }\end{array}$ \\
\hline septische Eingriffe & $\begin{array}{l}\text { Eingriffe bei bereits vorhandener Infektion oder nach Perforation } \\
\text { im Gastrointestinaltrakt. Bei dieser Wundkontaminationsklasse ist } \\
\text { das Operationsfeld schon präoperativ mit Erregern von möglichen } \\
\text { postoperativen Infektionen besiedelt. }\end{array}$ & $>20 \%$ & $\begin{array}{l}\text { Hohlorganperforation } \\
\text { mit Peritonitis }\end{array}$ \\
\hline
\end{tabular}

\section{Prinzipien}

\section{Prinzipien zur Optimierung der perioperativen} Infektionsprophylaxe mit Antibiotika

1. Eine interdisziplinäre Gruppe soll jährlich die PAP-Medikamente anhand lokaler Erreger-und Resistenzdaten festlegen. Eingriffe ohne Prophylaxe müssen klar definiert werden.

2. Die konkrete Applikation soll in die Hände der Anästhesie gelegt werden.

3. Die Gabe der Prophylaxe soll zuverlässig 30-60 min vor dem Eingriff erfolgen.

4. Die einmalige Gabe (Single Shot) ist bei einer Operationsdauer unter $3 \mathrm{~h}$ völlig ausreichend. Nur bei längerer Operation oder starkem Blutverlust sollte die Gabe wiederholt werden.

5. Eine über das Operationsende hinausgehende, verlängerte Prophylaxe ist nicht geeignet, die Zahl an Wundinfekten zu verringern und sollte unterbleiben.

\section{- Chirurgische Händedesinfektion}

Die Anwendung von Seife und Nagelbürsten sollte lediglich bei Arbeitsaufnahme oder bei sichtbarer Verschmutzung stattfinden. Die häufige Anwendung führt ansonsten zu vermehrter Hautreizung. Da bereits kleinste Einrisse Erregerreservoire bilden können, sollte Hautschutzcreme und bei individuellem Bedürfnis Hautpflegelotion angewendet werden. Für die Händedesinfektion vor dem operativen Eingriff wird eine Einwirkungszeit von 1,5 min bei folgendem Prozedere empfohlen [7]:

- zuerst Benetzung der Hände (10 s), dann der Unterarme (10 s) mit dem alkoholischen Präparat

- anschließende Händedesinfektionsphase (70 s): Benetzungslücken sind zu vermeiden, Hauptaugenmerk auf Fingerkuppen, Nagelfalze und Fingerzwischenräume

\section{- Begrenzung der Personenzahl und -bewegung im Operationssaal}

Bei Mischströmung steigt mit steigender Personenzahl und -bewegung im Operationssaal die mikrobielle Belastung der Raumluft im Bereich oberhalb des Operationstischs. Deshalb wird von der KRINKO eine Beschränkung der im Operationssaal anwesenden Personen empfohlen [8]. Auch der Einfluss von Türöffnungen hat bei orthopädischer Endoprothetik einen Einfluss auf die SSI-Rate. Die Relevanz für viszeralchirurgische Eingriffe bleibt aufgrund fehlender Studien allerdings offen. 


\section{- Operationsabdeckung}

Nach präoperativer Antiseptik wird das Operationsfeld mit als MP zertifizierten sterilen Abdeckmaterialien der Qualität „Standard“ oder „High Performance“ abgedeckt.

\section{- Screening des Operationsteams bei Ausbruch von SSI durch Staphylococcus aureus oder A-Streptokokken}

Bei Häufung von SSI ist ein Screening des Operationsteams im Vestibulum nasi durchzuführen.

\section{- Sterile Operationshandschuhe}

Es ist festzulegen, wann doppelte Handschuhe oder Handschuhe mit Perforationsindikator getragen bzw. ob bei Verzicht auf Double Gloving die Handschuhe während der Operation gewechselt werden. Letzteres ist bei mehrstündigen operativen Eingriffen sinnvoll. Nach dem Ablegen der Handschuhe empfiehlt sich die hygienische Händedesinfektion.

\section{Inzisionsfolie}

Bei Verwendung nicht antiseptisch imprägnierter Inzisionsfolie steigt das SSI-Risiko signifikant, deshalb ist ihr Einsatz kontraindiziert. Durch eine mit lod imprägnierte Folie wird die Wundkontamination verringert. Allerdings ist der Einfluss auf die SSI-Rate marginal.

\section{- Darmreinigung und orale Antibiose in der Darmchirurgie}

Durch die Kombination von Darmentleerung und oraler Antibiose wurden im Vergleich zum Verzicht auf beide Maßnahmen SSI, Ileus und Anastomosenleckage signifikant reduziert. Auch die alleinige Darmantibiose führte zur Reduktion von SSI und zu verkürzter Hospitalisierungsdauer, wenn auch in geringerem Ausmaß.

\section{nautversiegelung}

Die Versiegelung nach Hautantiseptik kann aufgrund widersprüchlicher Befunde derzeit nicht empfohlen werden.

\section{Intraoperative Präventionsmaßnahmen}

Die Tab. 7 zeigt die intraoperativen Maßnahmen zur Prävention von SSI im Überblick. Zur Erläuterung der Evidenzgrade siehe Tab. 4.

Aseptische Disziplin im Operationssaal. Ruhe, Vermeiden unnötiger Personenbewegungen, korrektes Tragen des Mund-Nasen-Schutzes, allseitige Bedeckung des Haares, sorgfältige Abdeckung des Patienten sowie Double Gloving und Handschuhwechsel bei Perforation tragen zur Minimierung des SSI-Risikos bei.

Keine ungeschützte Lagerung von Sterilgut. Die ungeschützte Lagerung von Sterilgut im Operationssaal außerhalb der Sterilverpackung bzw. die nachträgliche sterile Abdeckung auf dem Instrumententisch soll so kurz wie möglich erfolgen, sofern sich der Instrumententisch außerhalb des Lüftungsfelds einer Laminar-Air-FlowDecke befindet bzw. der Operationssaal mit turbulenter Mischströmung belüftet wird, weil Erreger aus der Umgebung auf den Instrumententisch aufgewirbelt werden können.

\section{Tabelle 7}

\section{Intraoperative Präventionsmaßnahmen.}

\begin{tabular}{|l|l|}
\hline Maßnahmen & Evidenzgrad \\
\hline Basishygiene & \\
\hline aseptische Disziplin im Operationssaal & IB \\
\hline $\begin{array}{l}\text { keine ungeschützte Lagerung von Sterilgut } \\
\text { außerhalb der Sterilverpackung }\end{array}$ & IB \\
\hline
\end{tabular}

fachspezifische Präventionsmaßnahmen

\begin{tabular}{|l|l|}
\hline Vermeidung akzidenteller Hypothermie & IA \\
\hline laparoskopische Operationstechnik & IB \\
\hline strenge Indikationsstellung für Drainagen & IB \\
\hline Operationstechnik und chirurgische Erfahrung & ॥ \\
\hline Wundretraktor & IB, II oder III \\
\hline antiseptisches Nahtmaterial \\
(Evidenz differiert indikationsabhängig)
\end{tabular}


Vermeidung akzidenteller Hypothermie. Intraoperative Hypothermie ist ein unabhängiger Risikofaktor für SSI. Daher soll perioperativ der Zustand der Normothermie aufrechterhalten werden, sofern nicht therapeutische Gründe eine Hypothermie erfordern.

Laparoskopische OP-Technik. Bei laparoskopischer Operation ist das Risiko sowohl für SSI als auch für Harnwegs- und pulmonale Infektionen aufgrund des kleineren Zugangs, früherer Mobilisation, geringerer Immunsuppression und geringerer Notwendigkeit zum Einsatz von ZVK signifikant geringer (Abb.4).

Strenge Indikationsstellung für Drainagen. Wunddrainagen sind ein unabhängiger Risikofaktor für SSI. Umgekehrt war keine Reduktion von SSI durch Drainagen nachweisbar. Daher sollen Wunddrainagen nur streng indiziert, so kurzzeitig wie möglich eingesetzt und über eine separate Inzision ausgeleitet werden. Offene Drainagen sind wegen des Infektionsrisikos nicht zu verwenden [9]. Die subkutane passive Drainage hatte einen günstigen Einfluss auf die SSI-Rate.

Operationstechnik und chirurgische Erfahrung. Atraumatisches Gewebehandling ist eine wichtige Voraussetzung für die primäre Wundheilung und damit für die Vermeidung von SSI. Chirurgen mit hoher Fallzahl jenseits der Lernkurve hatten bei minimalinvasiver Rektumchirurgie deutlich weniger SSI als Kollegen in der Lernkurve.

Wundretraktor. Durch Anwendung des Folien-Wundretraktors (Abb. 5) sollen im Inzisionsbereich die Feuchtigkeit aufrechterhalten und durch gleichmäßige Kräfteverteilung punktuelle Traumen vermieden werden. Zugleich wird durch den gleichmäßigen Zug an den Wundrändern weniger postoperativer Wundschmerz angegeben. Die Datenlage zum Einfluss auf SSI ist heterogen. In der jüngsten Metaanalyse konnte nach Laparotomie die infektionspräventive Wirkung bei kontaminierter, nicht jedoch bei sauber-kontaminierter und septischer Inzision gesichert werden. Zur Absicherung sind weitere Studien erforderlich.

Antiseptisch imprägniertes Nahtmaterial. Durch Imprägnierung mit Triclosan wird die Biofilmbildung auf dem Nahtmaterial unterbunden. Allerdings besteht eine Wirkungslücke gegen Pseudomonas aeruginosa. In $4 \mathrm{Me}-$ taanalysen wurde die Herabsetzung der SSI-Rate bei abdominellen Eingriffen bestätigt. Dagegen konnte in der PROUD-Studie bei elektiver medianer abdominaler Laparotomie kein signifikanter Einfluss auf SSI nachgewiesen werden. Nur das Auftreten einer Fasziendehiszenz, mög-

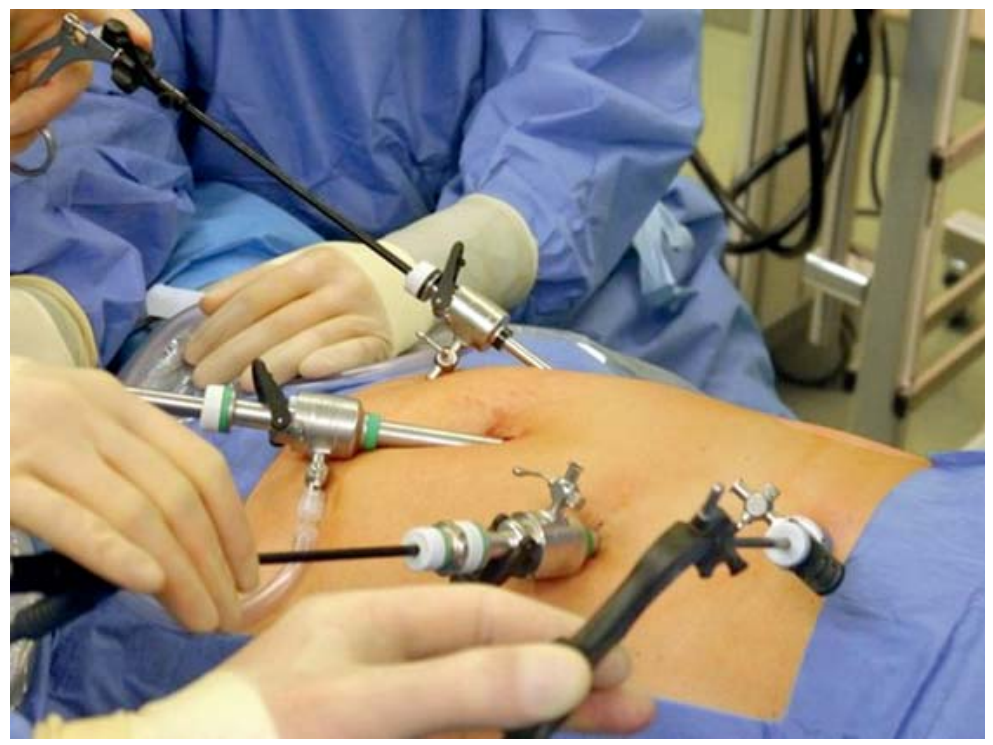

Abb. 4 - Laparoskopische Eingriffe gehen mit geringerem SSI-Risiko einher.

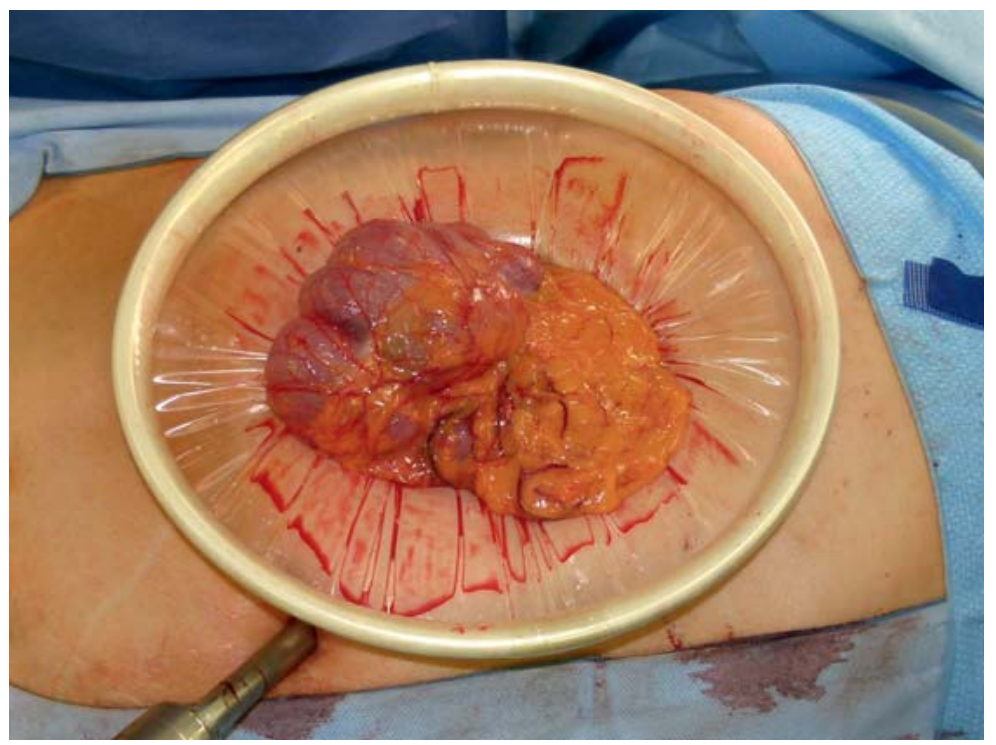

Abb. 5 - Folien-Wundretraktor.

licherweise Folge einer tiefen unentdeckten Wundinfektion, wurde signifikant reduziert. Bei Zusammenführung der Daten der PROUD-Studie mit 4 weiteren Studien ergab sich eine signifikante Überlegenheit zugunsten des antiseptischen Nahtmaterials. Tendenziell positive Ergebnisse wurden in der Brustkrebschirurgie und bei kardiochirurgischen Eingriffen erzielt. Bei gefäßchirurgischen Eingriffen an der unteren Extremität war kein Einfluss nachweisbar. Zur Absicherung bedarf es weiterführender Studien. 
Nahttechnik. Durch die Naht der Faszie mit kleinen Stichen konnte im Vergleich zu Big Bites eine Senkung der SSI-Rate erzielt werden. Zugleich erwies sich die Verwendung dünneren Fadenmaterials als günstig, evtl. als Folge der kleineren Oberfläche und der damit reduzierten Möglichkeit zur Biofilmbildung.

Antiseptische Spülung vor Wundverschluss. Durch intraoperative Wundspülung ist eine signifikante Reduktion der SSI-Rate erreichbar. In der Subgruppenanalyse war der stärkste Effekt hierzu bei kolorektaler Chirurgie nachweisbar. Welches der effektivste Wirkstoff in der klinischen Anwendung ist, ist noch zu klären.

\section{Postoperative Präventionsmaßnahmen}

Die Tab. 8 zeigt die postoperativen Maßnahmen zur Prävention von SSI im Überblick. Zur Erläuterung der Evidenzgrade siehe Tab. 4.

Desinfektion im Operationssaal. Nach der Operation eines Patienten mit Kolonisation oder Infektion mit MRE wird der Operationssaal bis zum Ablauf der Einwirkungszeit der Flächendesinfektion als „septisch“ deklariert, wobei auch das patientenferne Umfeld in die Zwischendesinfektion einbezogen wird. Das Operationsteam schleust sich neu ein und es erfolgt ein kompletter Wechsel der Reinigungsutensilien und der Bereichskleidung des Reinigungspersonals. Bei Verlegung auf die Station sind die KRINKO-Empfehlungen zur Isolierung einzuhalten $[10,11]$.

\section{Tabelle 8}

\begin{tabular}{|c|c|}
\hline \multicolumn{2}{|c|}{ Postoperative Präventionsmaßnahmen. } \\
\hline Maßnahmen & Evidenzgrad \\
\hline \multicolumn{2}{|l|}{ Basishygiene } \\
\hline Desinfektion im Operationssaal & IB \\
\hline Bettenhygiene & IB \\
\hline \multicolumn{2}{|c|}{ fachspezifische Präventionsmaßnahmen } \\
\hline aseptisches Wundmanagement & IB \\
\hline
\end{tabular}

\section{Hinweis}

\section{Leitlinie}

In der S1-Leitlinie „Anforderungen der Hygiene bei chronischen und sekundär heilenden Wunden“ [12] werden die Grundprinzipien der aseptischen Wundversorgung erläutert. Daher soll der Hinweis genügen, dass bei sekundär heilenden postoperativen Wunden die Indikationsstellung zur Wundantiseptik sorgfältig abgewogen werden muss, weil andernfalls die Wundheilung verzögert werden kann.

- Einmal täglich werden Verschmutzungen am Bett und Nachtschrank desinfizierend gereinigt. Nach Entlassung wird das Bett einschließlich Kopfkissen und Decke aufbereitet. Aus infektionspräventiven Gründen muss der frisch operierte Patient kein frisch bezogenes Bett bekommen.

Aseptisches Wundmanagement. Die Operationswunde wird am Ende der Operation mit einer sterilen Wundauflage versorgt. Der 1. Verbandswechsel erfolgt nach etwa 48 h, sofern nicht Hinweise auf eine Komplikation zu früherem Verbandswechsel Anlass geben. Ist die Wunde trocken und verschlossen, kann unter hygienischen Aspekten auf die erneute sterile Wundabdeckung verzichtet werden. Die regelmäßige ärztliche Inspektion der Wunde und die Befragung des Patienten auf Schmerzen im Wundbereich ist Teil der sachgerechten Nachsorge.

\section{Rahmenbedingungen}

Die Tab. 9 zeigt die Rahmenbedingungen zur Prävention von SSI im Überblick. Zur Erläuterung der Evidenzgrade siehe Tab. 4.

\section{Qualitätsmanagement der Hygiene und Surveillance.} Da ein relevanter Anteil von SSI durch Einhaltung von Hygieneregeln verhindert werden kann, muss von allen Verantwortungsträgern das Mögliche unternommen werden, um das Qualitätsmanagement der Krankenhaushygiene auszugestalten und durchzusetzen. Hierzu bedarf es klarer Regelungen mit fortlaufender Evaluation der Maßnahmen. 


\section{Hintergrund}

\section{Novellierung 2011}

Mit der Novellierung des Infektionsschutzgesetzes im Jahr 2011 wurden die Rahmenbedingungen zur Umsetzung der Krankenhaushygiene weiter verbessert und zugleich erhöhte Anforderungen an die Struktur- und Prozessqualität, sowie an Planungs- und Dokumentationspflichten gestellt, die Infektionsprävention sektorenübergreifend in Angriff genommen und Meldepflichten erweitert. Alle Gesundheitseinrichtungen sind zur Einhaltung der Infektionshygiene verpflichtet.

Die Ermittlung der Ergebnisqualität ist als prospektive Surveillance gesetzlich verpflichtend; dabei sind die Einrichtungen für das ambulante Operieren bei der Aufzeichnung und Bewertung bestimmter SSI den stationären Einrichtungen bereits seit 2001 gleichgestellt.

\section{Tabelle 9}

\begin{tabular}{|l|l|}
\hline Rahmenbedingungen. & \\
\hline Maßnahmen & Evidenzgrad \\
\hline $\begin{array}{l}\text { Qualitätsmanagement der Hygiene } \\
\text { und Surveillance }\end{array}$ & IA/IV \\
\hline Einführung eines SSI-Bündels & IB \\
\hline Fehleranalyse & $\|$ \\
\hline $\begin{array}{l}\text { Einbeziehung des Patienten in den } \\
\text { Infektionsschutz }\end{array}$ & $\|$ \\
\hline
\end{tabular}

Einführung eines SSI-Bündels. Zur Verbesserung der Compliance hat es sich als effektiv erwiesen, besonders wichtige Maßnahmen zu einem Maßnahmenbündel zusammenzufassen, dieses zu trainieren und die Einhaltung zu überwachen. Zweckmäßigerweise sollten 3-5 besonders wirksame Maßnahmen zu einem Bündel zusammengefasst werden, z.B. indikations-, zeit- und resistenzgerechte PAP, standardisierte präoperative Hautantiseptik, Normothermie und Surveillance. Dagegen bedarf die Einführung von Medizinprodukten wie Clipper, antiseptisches Nahtmaterial oder Wundretraktor außer der Entscheidung keiner Compliance.

\section{Hintergrund}

\section{Lernendes Team}

Ein lernendes Team ist am Eindruck der Patienten zur Einhaltung der Basishygienemaßnahmen im Betreuungsprozess durch Bewertung ausgewählter Hygieneindikatoren, z. B. mittels Fragebogen, interessiert. Die freiwillige, für Patient und Behandlungsteam anonymisierte Einbeziehung des Patienten als Monitor hat sich als praktikabel erwiesen, die Compliance des Behandlungsteams positiv zu beeinflussen. Allein durch die Frage nach der Durchführung der Händedesinfektion in vom Patienten nachvollziehbaren Situationen stieg der Verbrauch an Händedesinfektionsmittel um $15-40 \%$ an. Zugleich fühlen sich die Patienten ernst genommen. Durch die Möglichkeit des direkten Feedbacks des Patienten zur Einhaltung der Basishygienemaßnahmen in der Betreuung wird eine zusätzliche Sicherheitsbarriere für die Infektionsprävention aufgebaut.

Fehleranalyse. Wurden mehr als 2 Fehler bei der PAP (z.B. falscher Zeitpunkt, falsche Auswahl des Antibiotikums) festgestellt, hatte das signifikanten Einfluss auf die SSI-Rate. Allein durch die Kontrolle der Einhaltung der PAP mittels Checkliste war eine signifikante Senkung der SSI erreichbar.

\section{Einbeziehung des Patienten in die Infektionsprävention.} Selbst wenn die Gesundheitseinrichtung alle Präventionsmaßnahmen korrekt umsetzt, können der Patient und seine Angehörigen durch falsches Hygieneverhalten diese Bemühungen durchkreuzen. Deshalb ist es wichtig, den Selbstschutz des Patienten durch Vermittlung von Grundwissen zum Infektionsschutz zu fördern. Daher findet die Einbeziehung des Patienten und seiner Angehörigen in den Behandlungsprozess immer stärkere Beachtung. Gezielte Aufklärung (z.B. mittels Merkblatt, digitaler Informationsmaterialien, Patientenvideo) zu hygienegerechtem Verhalten während des Aufenthalts im Krankenhaus können das persönliche Gespräch nicht ersetzen, aber vor- und nachbereiten. 
Weiterführende Info

Relevante Institutionen für hygienische Belange

\section{Robert Koch-Institut}

Bundesinstitut für Infektionskrankheiten und nicht übertragbare Krankheiten. Aufgabe ist die Beobachtung des Auftretens von Krankheiten und relevanter Gesundheitsgefahren in der Bevölkerung als auch das Ableiten und wissenschaftliche Begründen der erforderlichen Maßnahmen zum wirkungsvollen Schutz.

\section{Kommission für Krankenhaushygiene und Infektionsprävention} des Robert Koch-Instituts

erstellt Empfehlungen zur Prävention nosokomialer Infektionen sowie zu

Maßnahmen der Hygiene in medizinischen Einrichtungen

\section{Kommission Antiinfektiva, Resistenz und Therapie} des Robert Koch-Instituts

Empfehlungen für Standards zu Diagnostik und Therapie von Infektionskrankheiten nach aktuellem Stand der medizinischen Wissenschaft

Deutsche Antibiotika-Resistenzstrategie des Bundesministeriums für 2020 wurde im Mai 2015 beschlossen. Ziel ist die Verhinderung der Verbreitung und Entstehung von Resistenzen sowie die Förderung von Forschung und Entwicklung zum Thema.

\section{Arbeitsgemeinschaft der Wissenschaftlichen Medizinischen}

Fachgesellschaften (AWMF)

koordiniert die Entwicklung von medizinischen Leitlinien für Diagnostik und Therapie durch die einzelnen wissenschaftlichen medizinischen Fachgesellschaften.

\section{Centers for Disease Control and Prevention}

Behörde der Vereinigten Staaten zum Schutz der öffentlichen Gesundheit. Ein wichtiges Aufgabengebiet der CDC sind Infektionskrankheiten, entspricht in etwa dem Robert Koch-Institut.

\section{Europäisches Zentrum für die Prävention} und die Kontrolle von Krankheiten

Ähnliche Institution auf europäischer Ebene. Aufgabenschwerpunkt ist die Beratung und Sammlung von Krankheitsvorkommen.

\section{Paul-Ehrlich-Gesellschaft}

bezweckt die Förderung von Forschung und Lehre auf den genannten Gebieten durch Erfahrungsaustausch, Abhaltung von wissenschaftlichen Veranstaltungen und gemeinschaftliche wissenschaftliche Untersuchungen.

\section{Krankenhaus-Infektions-Surveillance-System}

System zur systematischen Erfassung nosokomialer Infektionen des RKI, an dem Krankenhäuser freiwillig teilnehmen können.

\section{Schlussfolgerungen}

Analysen über Defizite bei Umsetzung evidenzgesicherter Standards unterstreichen, dass Basishygiene und die fachspezifischen Präventionsmaßnahmen unter Berücksichtigung ihrer Evidenz zu einer Multibarrierenstrategie zusammengeführt und umgesetzt werden müssen. Ausund Weiterbildung sowie Training im Arbeitsalltag sind dafür eine wichtige Voraussetzung. Dabei kommt der Surveillance von SSI einschließlich von MRE essenzielle Bedeutung zu, um aufgrund der Ist-Situation notwendige Interventionen ohne Zeitverzug einleiten zu können.

Bei allem Engagement ist zu berücksichtigen, dass sich Krankheitserreger an offensichtlichen wie an unerwarteten Lokalisationen finden können, denn es gibt weder das sterile Krankenhaus noch den nicht mikrobiell besiedelten Patienten und Besucher. Daher gibt es kein Nullrisiko für SSI, sondern nur eine Nulltoleranz gegenüber Hygienemängeln.

Die permanente Herausforderung besteht darin, jedes Teammitglied zu motivieren, anzuleiten und ggf. zu korrigieren, damit die Maßnahmen der Basishygiene und die fachspezifischen Präventionsmaßnahmen adäquat umgesetzt werden. Sicherheitskultur im Sinne des Infektionsschutzes bedeutet die schöpferische Umsetzung der Krankenhaushygiene durch fachkompetente, motivierte Mitarbeiter. Dabei kommt es darauf an, die Hygienestandards auf allen Verantwortungsebenen zu etablieren und ständig zu überarbeiten. Hygiene und Qualitätsmanagement sind unternehmenskritisch und Chefsache auf Krankenhausleitungsebene, Klinikebene, Abteilungsebene und Stationsebene. Dabei muss die Selbstverpflichtung zu einem optimalen Hygienemanagement von den Verantwortlichen konsequent vorgelebt werden.

Interessenkonflikt: Die Autoren geben an, dass kein Interessenkonflikt besteht. 


\section{Literatur}

1 Piening B et al. Nationales Referenzzentrum für die Surveillance von nosokomialen Infektionen. Deutsche Daten im Rahmen der ersten europäischen Prävalenzerhebung zum Vorkommen nosokomialer Infektionen und zur Antibiotikaanwendung. Epid Bull 2012; 26: $239-240$

2 Horan TC, Gaynes RP, Martone WJ et al. CDC definitions of nosocomial surgical site infections, 1992: a modification of CDC definitions of surgical wound infections. Infect Control Hosp Epidemiol 1992; 13: 606-608

3 Die Kategorien in der Richtlinie für Krankenhaushygiene und Infektionsprävention - Aktualisierung der Definitionen. Mitteilung der Kommission für Krankenhaushygiene und Infektionsprävention (KRINKO) beim Robert Koch-Institut (RKI). Bundesgesundheitsblatt Gesundheitsforschung Gesundheitsschutz 2010; 53: 754-756

4 Verordnung über das Errichten, Betreiben und Anwenden von Medizinprodukten (Medizinprodukte-Betreiberverordnung - MPBetreibV)

5 Anforderungen an die Hygiene bei der Aufbereitung von Medizinprodukten. Empfehlung der Kommission für Krankenhaushygiene und Infektionsprävention (KRINKO) beim Robert Koch-Institut (RKI) und des Bundesinstitutes für Arzneimittel und Medizinprodukte (BfArM). Bundesgesundheitsblatt Gesundheitsforschung Gesundheitsschutz 2012; 55: 1244-1310

6 Cruse PJ, Foord R. The epidemiology of wound infection. A 10-year prospective study of 62,939 wounds. Surg Clin North Am 1980; 60: 27-40

7 Händedesinfektion und Händehygiene. AWMF-Leitlinien-Register Nr. 029/027. HygMed 2015; 40-9: 369ff

8 Anforderungen der Hygiene bei Operationen und anderen invasiven Eingriffen. Mitteilung der Kommission für Krankenhaushygiene und Infektionsprävention (KRINKO) beim Robert Koch-Institut (RKI). Bundesgesundheitsblatt Gesundheitsforschung Gesundheitsschutz 2000; 43: 644-648

9 Prävention postoperativer Infektionen im Operationsgebiet. Empfehlung der Kommission für Krankenhaushygiene und Infektionsprävention (KRINKO) beim Robert Koch-Institut (RKI). Bundesgesundheitsblatt Gesundheitsforschung Gesundheitsschutz 2007; 50: $377-393$

10 Empfehlungen zur Prävention und Kontrolle von Methicillinresistenten Staphylococcus aureus-Stämmen (MRSA) in medizinischen und pflegerischen Einrichtungen. Empfehlung der Kommission für Krankenhaushygiene und Infektionsprävention (KRINKO) beim Robert Koch-Institut (RKI). Bundesgesundheitsblatt Gesundheitsforschung Gesundheitsschutz 2014; 57: 696-732

11 Hygienemaßnahmen bei Infektionen oder Besiedlung mit multiresistenten gramnegativen Stäbchen. Empfehlung der Kommission für Krankenhaushygiene und Infektionsprävention (KRINKO) beim Robert Koch-Institut (RKI). Bundesgesundheitsblatt Gesundheitsforschung Gesundheitsschutz 2012; 55: 1311 - 1354

12 Anforderungen der Hygiene bei chronischen und sekundär heilenden Wunden. AWMF-Leitlinien-Register Nr. 029/042, 2014

\section{Zum Weiterlesen und Vertiefen}

AWMF. Arbeitskreis Krankenhaus- und Praxishygiene der AWMF. Leitlinie: Perioperative Antibiotikaprophylaxe. AWMF-Register Nr. 029/022, Klasse: S1+IDA, 01/2012

Keenan JE, Speicher PJ, Thacker JKM et al. The preventive surgical site infection bundle in colorectal surgery. An effective approach to surgical site infection reduction and health care cost savings. JAMA Surg 2014; 149: $1045-1052$

Sutherland T, Beloff J, Lightowler $\mathrm{M}$ et al. Description of a multidisciplinary initiative to improve SCIP measures related to pre-operative antibiotic prophylaxis compliance: a single-center success story. Patient Saf Surg 2014; 8: 37

\section{Korrespondenzadresse}

Dr. med. Julius Pochhammer

Klinik für Allgemein-, Viszeral- und Thoraxchirurgie

Marienhospital Stuttgart

Böheimstraße 37

70199 Stuttgart

Telefon: 0711/6489-2201

Fax: $\quad 0711 / 6489-2213$

E-Mail: julius.pochhammer@vinzenz.de 


\section{Perioperative Medizin}

\section{CME-Fragen}

Welche Aussage zu Wund-

infektionen (SSI) trifft nicht zu?

\section{CME॰thieme.de}

\section{CME-Teilnahme}

- Viel Erfolg bei Ihrer CME-Teilnahme unter http://cme.thieme.de

- Bitte informieren Sie sich vorab online über die Gültigkeitsdauer.

- Sollten Sie Fragen zur Online-Teilnahme haben, unter http://cme.thieme.de/hilfe finden Sie eine ausführliche Anleitung.
Welche Aussage zur perioperativen Antibiotikaprophylaxe ist richtig?

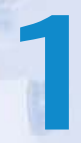

A Wundinfektionen sind nicht immer vermeidbar, da verschiedenste Einflussfaktoren eine Rolle spielen.

B Das Auftreten von SSI beeinflusst die Letalität nach abdominalchirurgischen Eingriffen nicht.

C SSI werden in oberflächlich, tief und organhöhlenbetreffend eingeteilt.

D Es gibt verschiedene Klassifikationen, die gebräuchlichste ist die der amerikanischen Centers for Disease Control and Prevention.

E Wundinfektionen erzeugen im Durchschnitt Mehrkosten für die Behandlung von ca. 3000 Euro.
Was ist kein Risikofaktor für die Entstehung postoperativer Wundinfektionen?
A Hauptziel der Prophylaxe ist die Vermeidung von intraabdominellen Abszessen.

B Der Zeitpunkt der Gabe der Prophylaxe ist nicht relevant.

C Die Prophylaxe sollte im Rahmen der Prämedikation verabreicht werden.

D Bei Patienten mit erhöhtem Risiko für eine postoperative Wundinfektion sollte die Prophylaxe noch 1-2 d länger gegeben werden.

E Bei erhöhtem Blutverlust und langer Operationsdauer ist eine erneute Gabe sinnvoll.
Welche Aussage zur Aufbereitung von Medizinprodukten trifft zu?
A Verzicht auf Darmspülung bei Dickdarmeingriffen

B Nikotinabusus

C Adipositas

D intraoperative Hypothermie

E Verzicht auf Ganzkörperwäsche vor der Operation
A Die Möglichkeit zur Aufbereitung ist durch den Hersteller sicherzustellen.

B Alle Medizinprodukte, die mit Blut und Schleimhäuten in Kontakt kommen, werden als kritisch eingestuft.

C Für die Aufbereitung aller Medizinprodukte der Kategorie „Kritisch“ ist eine Zertifizierung erforderlich.

D Nur bei externer Aufbereitung muss ein Qualitätsmanagement erfolgen.

E Grundlagen für die Aufbereitung von Medizinprodukten sind Regelungen im Sozialgesetzbuch V. 
Welche der folgenden Aussagen zu postoperativen Wundinfektionen trifft nicht zu?
A Eine postoperative Wundinfektion ist immer eine vermeidbare Komplikation.

B Allein die Erfassung von Wundinfektionen führt bereits zu deren Reduzierung.

C Ein unzureichendes Hygienemanagement wird nicht durch eine korrekt durchgeführte Antibiotikaprophylaxe kompensiert.

D Etwa 25\% der nosokomialen Infektionen sind Wundinfektionen.

E Wenn eine präoperative Haarentfernung erforderlich ist, ist das Clipping die beste Methode.
Welche Zuordnung von Wundklassifikation (nach Cruse) ist korrekt?
A Thyreoidektomie $\rightarrow$ bedingt aseptisch
B Cholezystektomie $\rightarrow$ bedingt aseptisch
C Abszessinzision $\rightarrow$ bedingt aseptisch
D Magenresektion $\rightarrow$ septisch
E Lungenresektion $\rightarrow$ aseptisch

Welches ist kein häufiger Erreger postoperativer Wundinfektionen?
7
A Escherichia coli
B Enterococcus faecalis
C Pseudomonas spp.
D Clostridium perfringens
E Staphylococcus aureus

Was muss bei der chirurgischen Händedesinfektion beachtet werden?
A Vor jeder Desinfektion müssen die Hände gewaschen werden.

B Achtung! Antwortformulierung weicht von Print-Version ab! Ideal sind alkoholbasierte Desinfektionsmittel ohne Zusatz von Chlorhexidin oder Octenidin.

C In Abhängigkeit von der mikrobioziden Wirksamkeit des Präparats ist eine Händedesinfektion von $30 \mathrm{~s}$ ausreichend.

D Hautschutz und -pflege hat weder einen positiven noch einen negativen Effekt auf die Wirksamkeit der Desinfektion.

E Zur Reinigung der Handinnenfläche sind sterile Einmalbürsten gut geeignet und sollten vorgehalten werden.
Welche Aussage zu Wunddrainagen ist nicht richtig?
A Zahlreiche Studien zeigen, dass die routinemäßige Drainageneinlage in vielen Fällen nicht sinnvoll ist.

B Drainagen sind Fremdkörper und stellen daher einen Risikofaktor für das Auftreten postoperativer Infektionen dar.

C Drainagen sollten so früh wie möglich entfernt werden.

D Idealerweise sollte auf eine gesonderte Inzision verzichtet werden.

E Achtung! Antwortformulierung weicht von Print-Version ab! Aufgrund des Infektionsrisikos sollten keine offenen Systeme verwendet werden.
Welche Aussage zur Berufskleidung ist korrekt?
A Für Tätigkeiten am Patienten (z. B. Verbandswechsel) sollte unbedingt der Arztkittel getragen werden, um den Kontakt mit den Unterarmen zu vermeiden.

B Das Tragen von Mund-Nasen-Schutz hat keine Auswirkung auf die Umgebungskontamination bei aseptischem Arbeiten.

c Private Oberbekleidung sollte in der Patientenversorgung nur unter dem Arztkittel getragen werden.

D Bereichskleidung sollte nur in dem vorgesehenen Bereich getragen werden, um die Verschleppung von Pathogenen zu vermeiden.

E Moderne sterile Operationshandschuhe sollen auch bei längeren Eingriffen nicht gewechselt werden, um Kosten zu sparen. 